\title{
DER SCHWEIZERISCHE MITTELSCHULATLAS* IN NEUER FORM
}

\author{
EduARD IMHoF
}

Im Frühjahr 1962 erschien der Schweizerische Mittelschulatlas in neuer Form. Von insgesamt 144 Kartenseiten mit 277 Karten wurden 101 Seiten mit 172 Karten oder $70 \%$ der Kartenteile des Werkes neu erstellt. 15\% der Karten wurden überarbeitet, und nur etwa $15 \%$ blieben im Inhalt im wesentlichen unangetastet. Veranlassung zu solcher Erneuerung boten unzählige Veränderungen auf der Erdoberfläche, neuc Forschungs- und Kartierungsergebnisse aller Art und in allen Erdräumen und nicht zuletzt auch tiefgreifende Neuerungen auf den Gebieten der Kartengestaltung und der Reproduktionstechnik. Hand in Hand mit den inhaltlichen, formalen und kartentechnischen Neuerungen wurde auch der methodische Aufbau des Werkes überprüft und wesentlich verbessert.

Der Schweizerische Mittelschulatlas enthält in seinen thematischen Karten weit mehr, als es in Schulatlanten oft üblich ist, Ergebnisse wissenschaftlicher Neu- und Originalbearbeitungen. Dies galt schon für die früheren Ausgaben, es gilt mehr noch für das heute vorliegende neue Werk. In der Kartengruppe Schweiz entstand nur die Niederschlagskarte durch bloßes Umformen einer bereits bestehenden Karte. Alle anderen thematischen Karten dieser Gruppe sind Ergebnisse früherer oder jüngster Neukonstruktionen. Diese Gruppe Schweiz ist daher gleichsam und in kleinem Rahmen ein Vorläufer eines geographischen Nationalatlasses unseres Landes.

Die Neuerstellung des Atlasses erstreckte sich über die Zeit vom Frühjahr 1956 bis zum Frühjahr 1962, gewisse Vorstudien und Vorarbeiten aber hatten schon im Jahre 1955 eingesetzt. Der gesamte Arbeitsaufwand, die zeichnerischen und reproduktionstechnischen Spezialstudien, die wissenschaftliche Bearbeitung der Karten, die Erstellung der Entwürfe, Reproduktionsoriginale und Druckplatten, umfassen eine Leistung von über 100000 Stunden. Hierbei sind administrative und verlegerische Arbeiten, Papier- und übrige Materialfabrikation, der Auflagedruck, die Erstellung des Einbandes, das Einbinden und die Atlasauslieferung nicht mitgezählt. Es mag wohl angezeigt sein, den neuen Atlas und einzelne Etappen seiner Erstellung hier einer Betrachtung zu unterziehen.

\section{DER INHALTLICHE AUFBAU}

Der Schweizerische Mittelschulatlas ist nicht das einzige, wohl aber das wichtigste Lehrmittel für den Geographieunterricht an unseren Gymnasien, Oberrealschulen, Handelsschulen und Lehrerbildungsanstalten. Die geographische Forschung und Lehre ist ungewöhnlich vielgestaltig und weitausholend, und das allgemeine Interesse an den stets wechselnden Schauplätzen des Weltgeschehens ist außerordentlich groß. Die Anzahl der Schuljahre und Schulstunden aber bleibt notgedrungen begrenzt. Jeder Geographieunterricht, und sei er noch so klug und systematisch aufgebaut, ist daher selektiver Art. Der Lehrer trifft eine Auswahl der Betrachtungsgebiete, und er wird dabei in der Regel vom Nahen, Bekannten, vom unmittelbar Sichtbaren Schritt für Schritt vorrücken zu fernen Gebieten, deren Kenntnis nur durch Karte, Bild und Beschreibung vermittelt werden kann. Er wird durch geschickte Wahl der Gebiete und Gegenstände in die wichtigsten natur- und kulturgeographischen Erscheinungen und schließlich in ihre vielfältigen Zusammenhänge und Verflechtungen einführen. Im Gegensatz etwa zur elementaren Geometrie oder Algebra oder Sprachlehre ist es auf länderkundlichem Gebiete schwierig, ein allgemein zusagendes, kurzgefaßtes Lehrbuch zu schaffen. Nicht leichter ist dies für einen Schulatlas; denn ein solcher ist naturge-

* Ed. Imнof : Schweizerischer Mittelschulatlas, 13. Auflage. Zürich: Kantonaler Lehrmittelverlag, 1962. - Ed. IMHof: Atlas scolaire suisse pour l'enseignement secondaire, 12e éd. Lausanne: Editions Payot, 1962. - Ed. ImHoF: Atlante Svizzero per le scuole medie, 8a ed.Zurigo: Kantonaler Lehrmittelverlag, 1962. 
mäß stets nur Stoffsammlung, nie aber entspricht sein Inhalt einem bestimmten, von A bis $Z$ durchzuarbeitenden Lehrgang.

Die Gesamtpläne früherer Auflagen des Atlasses waren unter Mithilfe der erfahrensten Geographen und Geographielehrer des Landes entwickelt worden. Es stellte sich uns nun die Frage nach einer Revision des bisherigen, im ganzen gesehen bewährten und systematischen Gesamtplanes. Eine Befragung zahlreicher Geographielehrer ergab zwar eine Menge guter Anregungen und Ergänzungswünsche, kaum aber grundsätzlich neue methodische Gesichtspunkte. Wohl hat sich die länderkundliche Forschung im Laufe der letzten Jahrzehnte stark gewandelt, die analysierende Betrachtung physiogeographischer und kulturgeographischer Dinge hat sich mehr und mehr der vergleichenden Betrachtung, dem Studium der gegenseitigen Verflechtungen der Dinge in der Landschaft, zugewendet. Solchen Wandlungen der Forschung vermag aber der Geographieunterricht nur zögernd zu folgen; denn er kann auf eine primäre Vermittlung grundlegender Kenntnisse nicht verzichten. Auch die Karte kann nicht gleichzeitig Analyse und Synthese bieten. Die Synthese ergibt sich hier in der Regel als eine geistige Erkenntnis aus der gleichzeitigen und vergleichenden Betrachtung einer Reihe von Karten ein und desselben Gebietes, jedoch verschiedenen Inhaltes. Solche Kartenreihen aber bot der Schweizerische Mittelschulatlas bereits in der bisherigen Form. In gewissem, eingeschränktem Sinne ist jede gute topographische Karte bereits eine synthetische Darstellung. Eine vollsynthetische, alle wesentlichen geographischen Elemente zusammenfassende und die daraus resultierenden neuen Vereinigungsgefüge zeigende Karte ist bis zur Stunde in brauchbarer Form nicht entwikkelt worden. Trotz solcher Vorbehalte zeigte sich aber bei der Revision des Inhaltsplanes unseres Atlasses folgende Möglichkeit einer Anpassung an die neueren Betrachtungsweisen:

Bisher wurden die Schweiz und jeder Erdteil in einer sogenannten «physischen» wie auch in einer gleich großen «politischen» Karte dargestellt. Diese Aufspaltung der Inhaltselemente auf zwei Karten war längst überfällig. Jedes dieser Gebiete erscheint nun in einer einzigen, die natürlichen und die kulturellen Erscheinungen kombinierenden Karte, die Schweiz im Maßstab 1:1000 000, die Erdteile in 1:30000 000. Die staatlichen Gliederungen aber sind, wie andere thematische Spezialinhalte, in kleineren Nebenkarten nochmals beigefügt.

Durch solche und andere Umstellungen und durch einige Weglassungen weniger wichtiger Dinge konnte beträchtlicher Raum gewonnen werden. Damit konnten zahlreiche neue Karten aufgenommen und bestehende Karten vergrößert werden, ohne die Anzahl der Seiten vermehren zu müssen. Die Einhaltung des bisherigen Gesamtumfanges war vom Auftraggeber gewünscht worden, weil man eine periodisch wiederkehrende Erhöhung der Druckkosten vermeiden wollte. Bisher gelangten viele Gebiete nur in den Erdteilkarten, d.h. im Maßstab 1:30000 000 zur Darstellung. Nun erscheint jedes wichtigere Gebiet mindestens im Maßstab 1:15000 000. Darin liegt ein beträchtlicher Gewinn.

In größeren Maßstäben dargestellt als bisher wurden folgende Gebiete:

Skandinavien 1:6000 000, bisher 1:8000000 (S. 70-71)

Nildelta und Suezkanal 1:2000 000, bisher 1:4000 000 (S. 100)

Titicaca-Becken 1:7 500000 , bisher 1:10000 000 (S. 120)

Polargebiet 1:40000 000, bisher 1:60000000 (S. 134-135)

Neu aufgenommen wurden Karten folgender Gebiete:

Gruyères (Kt. Freiburg) (S. 2)

Mürtschenstock (Kt. Glarus) (S. 2)

Matterhorn und Z'Muttgletscher (S. 3)

Karstlandschaft bei Postojna (S. 49)

Marmarameer (S. 49)
Spitzbergen (S. 69)

Island (S. 70)

Donaumündung ( $\mathrm{S} .74$ )

Mount Everest (S. 93)

Ostafrika (S. 105) 
Südafrika (S. 105)

Hawaii-Inseln (S. 113)

Grand Canyon des Colorado (S. 113)

Mississippi-Delta (S. 113)
Kolumbien und Venezuela (S. 120)

Südbrasilien und La Plata-Länder

(S. 121)

Südamerika, südlicher Teil (S. 121)

Im Gebietsumfang wesentlich erweitert wurden folgende Karten:

Stadt Zürich (S. 11)

Südostasiatische Inseln (S. 94-95)

Ostasien (S. 90)

Jordangraben (S. 101)

sowie einige kleinere Karten (Ätna, Moskau usw.).

$\mathrm{Zu}$ dieser Bereicherung des allgemeinen «topographischen» Atlasteiles gesellt sich eine ebenso große Menge größer dargestellter oder neu eingefügter thematischer Karten. In größeren Maßstäben als bisher wurden folgende thematische Karten dargestellt:

Europa Vegetation 1:30000 000, bisher 1:40000 000 (S. 77)

Europa Wirtschaft 1:22 500 000, bisher 1:25000 000 (S. 83)

Nordamerika: Industrie und Fischerei, Produkte des Landbaues und Bergbau. Alle diese drei Karten in 1:35000 000, bisher 1:45000 000 (S. 116-117)

Erde: Politische Gliederung und Flug- und Schiffslinien 1:100 000 000, bisher

$1: 170000000$ (S.138)

Neu aufgenommen wurden folgende thematische Karten (wobei neue Karten, welche bisherige Karten desselben oder eines ähnlichen Themas ersetzen, nicht mitgezählt sind) :

Italien und Balkan: Eine zweite Isothermenkarte, so daß nun die Januar- und die Juli-Isothermen getrennt zur Darstellung kommen (S. 52).

Italien: Zwei neue Karten: Winter- und Sommerniederschläge (S. 53).

Iberische Halbinsel: Zwei neue Karten für Januar- und August-Isothermen (S.61).

Skandinavien: Eine zweite wirtschaftsgeographische Karte (S.69).

Europa: Geologie (S. 73).

Südostasien: Zwei neue Karten: Niederschläge im Januar und im Juli (S. 85).

Asien: Fünf neue Produktekarten von Teilgebieten (S. 88, 91, 92, 93 und 95).

Australien und Neuseeland: Neu eine Karte der Tektonik. Ferner zwei wirtschaftsgeographische Karten an Stelle einer einzigen (S. 129).

Für die Erdübersichten der Volksdichte, der politischen Gliederung, der Weltwirtschaft und des Weltverkehrs, der Völker, Religionen und der Produkte wurde eine geeignetere, die Randgebiete weniger verzerrende, flächentreue Projektion gewählt, diejenige von Hammer-Wagner mit verlängerter Pollinie. Damit gelang es, trotz beträchtlicher Raumeinsparung bei der Weltwirtschaftskarte, die interessierenden Gebiete gleich groß und zum Teil größer als bisher darzustellen.

Diesen Gewinnen stehen einige Verluste gegenüber, jedoch nur solche, die offenbar verschmerzt werden können. Es sind folgende (die Seitenzahlen beziehen sich hier auf die Atlas-Auflage 1958):

S. 2 und 3: Beispiele aus den amtlichen eidg. Landeskarten, ersetzt durch neue Beispiele.

S. 49: Kleinkärtchen von Istanbul und Bosporus, ersetzt durch neue Karte des Marmarameeres.

S. 49: Bucht von Kotor, ersetzt durch eine neue, kleinere Karte.

S. 49: Kleinkärtchen von Athen wurde weggelassen, weil das wachsende Häusermeer die verfügbare Kartenfläche überbordete.

S. 56: Anlandung von Inseln bei Piombino-Orbetello, ersetzt durch eine Karte eines Teiles dieses Gebietes. 
S. 63: Kleinkärtchen von Barcelona, Madrid und Lissabon eliminiert.

S. 70: Kleinkärtchen von Leningrad eliminiert. Es genügt hier die Karte der Newabucht.

S. 70: Limanküste bei Odessa. Weil keine genügenden topographischen Grundlagen ererhältlich, wurde dieses Kärtchen ersetzt durch ein solches der Donaumündung. Dieses Kärtchen zeigt den Seentypus der Limans ebenfalls.

S. 88-89: Die Wirtschaftskarte von Asien (S. 92) wurde wesentlich kleiner dargestellt, was durch bessere graphische Gestaltung und durch geringfügige Vereinfachungen ermöglicht wurde, ohne Inhalt und Lesbarkeit der Karte zu schmälern. Damit wurde nicht nur viel Raum gewonnen, sondern diese Karte maßstäblich den Karten der Vegetation und der politischen Gliederung desselben Gebietes angeglichen.

S. 94-95: Die Karte China-Japan 1:10000 000 ist ersetzt durch die Karte Ostasien 1:15000 000. Damit konnte das Gebiet nach Norden erweitert, maßstäbliche Übereinstimmung mit den Karten von Indien, vom Orient usw. erreicht und gleichzeitig der Raum einer halben Seite gewonnen werden.

S. 97: Asien Produkte ist ersetzt durch fünf neue Produktekarten wichtiger Teilgebiete.

S. 100: Die Karte Israel und Jordanien 1:1000 000 ist ersetzt durch eine neue Karte des Jordangrabens 1:2000 000. Damit wurde eine Erweiterung auf das ganze Gebiet des Jordangrabens bis zu dessen Fortsetzung im Golf von Akaba und ein unmittelbarer räumlicher Anschluß an die Karte des Nildeltas ermöglicht (S. 100101 des neuen Atlasses).

S. 106: Das Kleinkärtchen von Kairo wurde eliminiert (siehe größere Karte des Nildeltas).

S. 124: Karte von Rio de Janeiro, auf ein engeres Gebiet beschränkt. Karten der Magallanstraße und des Rio de la Plata, ersetzt durch neue Karten größerer Gebiete in kleineren Maßstäben.

S. 129: Kleinkärtchen von Sydney und von Canberra eliminiert.

S. 134 und 135: Miniaturkärtchen der Land- und Wasserhalbkugeln weggelassen.

S. 136: Erdkarte der Malariagebiete eliminiert, jedoch die Malariagrenzen in die Volksdichtekarte der Erde aufgenommen.

Wie diese Zusammenstellung zeigt, wurden vorwiegend einige Gebietskleinkärtchen weggelassen, besonders solche ausländischer Städte. Damit folgte der Atlasredaktor einem aus Kreisen der Geographielehrer mehrfach geäußerten Wunsche. Die Erfahrung zeigte, daß Kleinkärtchen von Städten, Karten in zu kleinen Maßstäben und mit zu enger Gebietsabgrenzung, im Unterricht nicht befriedigend ausgeschöpft werden können.

Zusammenfassend kann festgestellt werden, daß die Karten wesentlich vermehrt und planvoller ausgewählt worden sind. Bei der Auswahl der allgemeinen «topographischen» Karten wurde darauf geachtet, daß neben wichtigsten, dichtest bevölkerten Gebieten vor allem alle in der Schule zu behandelnden Landschaftstypen (Gebirgsformen, Talformen, Ebenenformen, Küstenformen, Gletschertypen usw., aber auch Siedlungsformen, Meer- und Flußhäfen, Flughäfen usw.) gut vertreten sind. Durch die thematischen Karten sollen Gebiete von ähnlicher Bedeutung möglichst gleichwertig, durch gleichartige oder ähnliche Reihen von Karten zur Darstellung gelangen, und es sollen überdies regional besonders charakteristische Dinge gezeigt werden. In diesem Sinne wurde die Auswahl da und dort verbessert. Reicher ausgestattet wurden besonders die Geologie, die Klimatologie, die Wirtschaftsgeographie und die Verkehrsgeographie. 


\section{DIE QUELLENBESCHAFFUNG}

Über 10 Millionen Bauleute sind jeden Tag damit beschäftigt, das Antlitz der Erdoberfläche umzuformen. Auch die politischen Verhältnisse, die soziologischen $\mathrm{Zu}$ stände und die Ortsnamen sind überall andauernden Wandlungen unterworfen. Dazu treten die Umgestaltungen durch die Kräfte der Natur. Wohl über 50000 Menschen Geometer, Topographen, Statistiker, Naturwissenschafter, Geographen, Flugphotographen, Kartographen, Drucker usw. und ihre Gehilfen - arbeiten an der Erforschung und Abbildung all der Zustände und ihrer Veränderungen. Die Ergebnisse solchen Geschehens, Forschens und Kartierens, selbst die wichtigsten und großräumigsten Veränderungen, gelangen nicht sofort und «gebrauchsfertig» auf den Tisch des Atlasredaktors. Das unübersehbare, weit verstreute Material von Plänen, Karten und Zählungsergebnissen wird durch unzählige Kanäle geschleust, vom Urmaterial zu konzentrierteren Zusammenfassungen, von detailreichen Aufnahmekarten zu schrittweise abgestuften Folgekarten kleineren Maßstabes. Dieses Durchschleusen und Durchsieben ist nur bezüglich einzelner weniger Staatskartenwerke planmäßig organisiert, großenteils unterliegt es dem Zufall, dem Willen und der Tätigkeit einzelner Forscher und Forschergruppen, einzelner Verleger, Kartographen usw. So kommt es, daß einzelne neue topographische Veränderungen oder neue wissenschaftliche Daten schon wenige Monate nach ihrer örtlichen Feststellung dem Atlasredaktor «griffbereit» vorliegen, während andere Dinge erst nach Jahren, ja oft erst nach Jahrzehnten zur Verfügung stehen. Eine Zeitungsnotiz über die Eröffnung einer neuen Bahnstrecke bei Ulan-Bator oder über das Auffüllen eines neuen Stausees in Halungkiang hilft nichts; denn Zeitungsberichte sind oft Zeitungsenten. Dem Kartographen hilft nur eine einwandfreie Zeichnung eines Objektgrundrisses. Diese aber versteckt sich oft noch jahrelang hinter den sieben Bergen, selbst wenn ein Gerücht schon längst siebenmal um die Welt gezittert ist. Jedes menschliche Tun, auch jedes kartographische Tun ist mit Fehlern behaftet. Der Geodät «liebt» vor allem die zufälligen und die systematischen Fehler, der Kartograph aber die ganz groben. Diese schleichen sich ein in jedes Dokument, und sie besitzen eine außerordentliche Zeugungskraft. Sie pflanzen sich fort und verseuchen dann oft jahrzehntelang Hunderte von Folgekarten. Diese Untugend der Kartographie muß der Atlasredaktor stets vor Augen haben.

Der Atlasbenützer aber verlangt «moderne», nachgeführte, «richtige» Karten. Was tun? Es bleibt nichts anderes übrig, als mit einfachsten, aber gut ausgedachten Mitteln einen geographischen Suchdienst aufzubauen. Hauptwerkzeuge hierfür sind gute Kenntnisse über bibliographische und bibliothekarische Hilfsmittel. Einige Andeutungen über dieses wichtige und zeitraubende Teilgebiet einer Atlaserstellung mögen auch hier interessieren.

Beim Schweizerischen Mittelschulatlas handelt es sich um einen «komplexen Weltatlas». Der Atlas enthält, wenn auch meist nur in kleinen Maßstäben und in stark vereinfachten Formen, topographische und thematische Karten aller Gebiete der Erde. Entsprechend hatte auch unser geographischer Suchdienst fast alle Gebiete der Erde und viele verschiedene Themen zu erfassen.

Hauptquellen für die Erstellung eines Schulatlasses sind sogenannte Handatlanten; denn sie enthalten ein gewaltiges topographisches Grundmaterial bereits in gesiebter, vereinfachter, verkleinerter und zusammengefaßter Form. Bestgeeignete Basiskarten für die Erstellung einer neuen Karte sind solche zwei- bis fünfmal größeren Maßstabes. Karten gleichen Maßstabes sollten nur in Ausnahmefällen beigezogen werden, da man ihren individuellen Generalisierungseigenarten ausgeliefert ist. Die als Quellen beizuziehenden Handatlanten sind jedoch sorgfältig auszuwählen. Oft enthalten die als neu angepriesenen Atlanten alte Karten, die nur notdürftig nachgeführt sind. Nur alle paar Jahrzehnte einmal wird durch irgend einen kartographischen Großverlag ein guter und fundamental neuer Handatlas in die Welt gesetzt. Karten solcher 
Atlanten bilden jeweils unmittelbare Vorlagen für ungezählte kleinere Werke, für Schulatlanten und dergleichen. Vor und nach der letzten Jahrhundertwende kam die Ehre des meist ausgeplünderten Atlasses vor allem dem «Stieler» aus Justus Perthes Geographischer Anstalt in Gotha zu. Er war auch die Hauptquelle des ausländischen topographischen Kartenteiles der ersten Ausgabe des Schweizerischen Mittelschulatlasses vom Jahre 1910. Für die von mir in den Jahren 1927-1932 besorgte Umformung des Mittelschulatlasses war es der damals sensationelle «Grande Atlante del Touring Club Italiano». Für einen Teil der Karten der jüngsten Ausgabe, derjenigen des Jahres 1962, erwiesen sich als besonders reiche Quellen der russische Atlas «Mira» (Moskau 1954) und «The Times-Atlas of the World» (Mid-Century Edition, edited by John Bartholomew, Vol. 1-5, London 1955-1959). In Zukunft wird auch der 1961 erschienene «Große Bertelsmann» (Verlag Bertelsmann in Gütersloh) ausgezeichnete Dienste leisten. Zur Ergänzung wird man stets auch kleinere Atlanten, Schulatlanten, Schulkarten und andere Übersichtskarten beiziehen. Grundsätzlich entnehmen wir jedoch einem polnischen Atlas nur Daten polnischer Gebiete, einem schwedischen Atlas nur solche über Schweden, einem belgischen Atlas nur solche über Belgien usw.; denn die Geographen und Kartographen eines jeden Landes kennen ihr eigenes Land am besten, und sie kommen dortigen Veränderungen am raschesten auf die Spur. Grobe Fehler und Vernachlässigung werden bei Darstellungen des eigenen Landes selten begangen. Fremdes Gebiet aber wird oft fehlerhaft, lückenhaft und in veralteter Form kartiert. Für Gebietskarten größeren Maßstabes, wie z.B. für unsere vielen Städte- und Landschaftskarten 1:500000 und größer, mußten - soweit erhältlich - die jüngsten amtlichen topographischen Blätter der fraglichen Gebiete benützt werden. Aber auch diese sind oft nicht genügend nachgeführt. Der Atlasredaktor war daher weitgehend auf persönliche Auskünfte ausländischer Spezialkenner angewiesen. Wir erfreuten uns der freundschaftlichen Hilfe solcher Leute aus den verschiedensten Ländern und in reichstem Maße.

$\mathrm{Zu}$ den unentbehrlichen Quellen für unsere Gebietskarten kleiner Maßstäbe zählen auch einige internationale statistische Jahrbücher, vor allem die folgenden:

1. The Statesman's Year-Book, London

2. Jahrbuch der Welt. Hrsg.: Karl Wagner, München

3. Statistiken der Gemeinde-Einwohnerzahlen verschiedener Staaten.

4. Dictionnaire des Bureaux de Poste. Hrsg.: Bureau International de l'Union Postale Universelle, Bern.

Besonders schwierig ist die Beschaffung genügender kartographischer Unterlagen zur Gestaltung der Reliefformen gewisser fremder Gebiete. Die neue Reliefdarstellung der Atlasausgabe 1962 vermag die Oberflächenformen viel genauer und detailreicher wiederzugeben, als es seinerzeit mittelst der steifen und manierhaften Schraffengefüge möglich war. Für viele Gebiete aber bestehen heute noch keine genügend genauen und genügend anschaulichen topographischen Karten oder sie werden geheimgehalten.

Noch weiter zurück aber ist die thematische Kartographie. Selbst für das Gebiet der Schweiz, diesen landkartengeschwängerten Erdstrich, wurden seit einem halben Jahrhundert keine neuen Karten der Januar- und der Juli-Isothermen, der relativen Volksdichte mit geographischer Abgrenzung und der Gruppierungen der Landessprachen herausgebracht. Alle seither irgendwo publizierten Karten solcher Art entstammten inhaltlich der Ausgabe 1910 des Schweizerischen Mittelschulatlasses. Unter solchen Umständen ist es sehr zu begrüßen, daß seit einigen Jahrzehnten in einigen Ländern geographische Landes- oder Nationalatlanten, ferner Länder- und Weltatlanten der Klimatologie, der Wirtschaft usw., herausgegeben werden. Durch solche meist groß angelegten Werke erfuhr die geographisch-thematische Kartographie einen außerordentlichen Auftrieb. Für die Neuerstellung oder Bereinigung der thematischen Karten des Mittelschulatlasses standen uns bereits etwa ein Dutzend solcher Groß- 
atlanten zur Verfügung. Das meiste neuere Material thematisch-kartographischer Art aber mußte den verschiedensten Einzelkarten und Zeitschriftenaufsätzen aus aller Welt entnommen werden, oder aber die Karten mußten durch uns und unsere Mitarbeiter inhaltlich neu geschaffen werden. Dieses letztere trifft für fast alle Karten der Schweiz zu, ferner für die Karten der Vegetation, der Wirtschaft, der Völker, der Religionen aller Gebiete.

Die Beschaffung eines solch weitschichtigen und stetsfort weiterwachsenden und sich wandelnden Grundmaterials wäre ohne den Beizug der Kartenbestände des Kartographischen Institutes der Eidg. Technischen Hochschule und ohne die Mithilfe weiterer Bibliotheken kaum möglich gewesen.

Trotz all unserer Bemühungen ist es nicht zu vermeiden, daß zum Beispiel fünf Monate nach der Fertigstellung des Auflagedruckes der Balkankarten unseres Atlasses ein nagelneuer rumänischer Nationalatlas herausgegeben wird, dessen Inhalt unsere Isothermen in Siebenbürgen Lügen straft!

\section{DIE ALLGEMEINEN LÄNDER- UND ERDTEILKARTEN}

Die allgemeinen Gebietskarten kleinerer Maßstäbe - wir zählen sie, ihrem Inhalt entsprechend, im weitesten Sinne zu den topographischen Karten - erscheinen in der Atlasausgabe 1962 erstmals in neuer Gestalt. Zum erstenmal in einem Schulkartenwerk gelangte dabei eine vom Atlasredaktor entwickelte neuartige Geländedarstellung zur Anwendung. Wir treten hier nur kurz auf diese Kartenform ein, da wir sie in der Geographica Helvetica (Lit. Nr. 4) bereits eingehend erläutert hatten.

Die früher üblichen abstrakten Schraffen und die konventionellen Höhenstufenfarben wurden ersetzt durch eine unmittelbar formen- und höhenplastische Darstellung. Dies führte zu einer weniger manierhaften Gestaltung der Reliefformen und zu ruhigeren und anschaulicheren Kartenbildern. Diese neue Darstellungsform ermöglicht ein genaueres, detailreicheres Erfassen der Geländeformen. Als Grundlagen für ihre zeichnerische Gestaltung sind daher, wie bereits betont, wesentlich genauere Basiskarten erforderlich, als es für die einstigen manierhaften Schraffengebirge der Fall gewesen war. In der Beschaffung solcher Basiskarten liegt bis auf weiteres eine beträchtliche Schwierigkeit. Überdies gibt es nicht viele Kartographen, die mit ausreichender zeichnerischer Fähigkeit genügenden Sinn für Erdoberflächenformen vereinigen, um gute, möglichst manierfreie Relieforiginale erstellen zu können. Glücklicherweise gelang es dem Atlasredaktor immer wieder, tüchtige Leute zu finden, die sich in die neuen Methoden einführen ließen.

Heute versuchen amerikanische, englische und schwedische Kartographen in Reliefkarten, besonders in solchen kleiner Maßstäbe, die Höhenstufenfarben durch naturähnliche Erdoberflächenfarben, durch die landschaftlich-farblichen Aspekte des Kulturlandes, der Wälder; Steppen, Wüsten usw. zu ersetzen (Lit. Nr.6). Dies ist ein lobenswertes, aber nicht neues Bemühen. Schon Prof. Fridolin Becker (Linthal und Zürich, 1853-1923), einer der Begründer der Reliefkartographie, malte Kartenbeispiele solcher Art. Erinnert sei an seine Karte des Rigi 1:50 000, erschienen um 1900 in Winterthur, ferner an die Karte «Jerusalem und Mitteljudäa» 1:100 000, erschienen 1914 in Bern. Seither wurde dieser Weg in der Schweiz und anderswo immer wieder beschritten, jedoch vorwiegend bei der Erstellung von Karten großer Maßstäbe. Auch die Karte «Jungfraugruppe und Aletschgletscher» 1:100 000 auf Seite 6 und diejenige des Grand Canyon des Colorado 1:250 000 auf Seite 113 des hier besprochenen Atlasses können als Beispiele hierfür gelten, ebenso die 1952 erschienene «Carte de la Chaîne du Mont Blanc au 10 000me» des Institut Géographique National in Paris. Ein mit allen malerischen Mitteln konsequent durchgeführtes, aber bisher nicht veröffentlichtes Beispiel ist das vom Verfasser im Jahre 1938 erstellte große Kartenoriginal des Walenseegebietes 1:10 000 im Kartographischen Institut der Eidg. 
Techn. Hochschule. Zur farbigen Gestaltung der oben genannten Karte des Grand Canyon im Mittelschulatlas 1962 dienten als Vorlagen farbige Luftbilder, die der Verfasser anläßlich eines privaten Fluges über jenes großartige Felsental im Jahre 1958 aufgenommen hatte.

Die Wiedergabe der natürlichen Landschaftsfarben in Reliefkarten kleiner und sehr kleiner Maßstäbe ist nun freilich ein interessantes und sohwer lösbares Problem. Noch fehlen uns für die meisten Gebiete der Erde die hierzu benötigten farbigen Luftbilder. Überdies besitzen alle niederschlagsreichen Gebiete der Erde während eines großen Teiles des Jahres uniforme grüne Landschaftsfarben, so daß bei deren naturähnlicher Wiedergabe keine genügenden farblichen Gliederungen erreicht würden. $\mathrm{Zu}$ anderen Jahreszeiten aber ist das Mosaik des farbigen Luftbildes vielenorts derart bunt gefleckt, daß bei dessen Übertragung auf Karten kleinen Maßstabes jeglicher Reliefeindruck zerhackt oder mindestens äußerst stark gestört würde. Nur in der Trockenwüste und in der Schneelandschaft ist die Oberfläohe farbig so uniform und im allgemeinen so hell, daß im Abbild von oben bei günstigem Sonnenstand durch Licht und Schatten gute plastische Effekte entstehen. Die Versuchsbeispiele landschaftsfarbiger Karten zeigen daher vorzugsweise aride Gebiete (Kartentafel in Lit. Nr.6).

Je kleiner der Maßstab, desto feingliedriger das Mosaik landschaftsähnlicher Farben und desto gestörter die Reliefeffekte. Um solche Schwierigkeiten zu verringern, werden bei entsprechenden Darstellungen in Karten sehr kleiner Maßstäbe die Landschaftsfarben meist nur in gröbster Zusammenfassung gegeben, etwa entsprechend den Flächengliederungen von Karten der Vegetationszonen. Beispiele dieser Art enthält der «Life Pictorial Atlas of the World» (Editors of Life and Rand Mc. Nally. New York 1961). Solche summarische Flächengliederungen mit ihren fließenden Übergängen entfernen aber die Karten von der angestrebten Naturähnlichkeit. Farbige Naturähnlichkeit, d.h. ein Angleichen der Karten an das farbige Luftbild, ist für gewisse $Z$ wecke zweifellos ein erstrebenswertes Ziel. Karten solcher Art besitzen neben anderen Darstellungen ihre Berechtigung. Sie vermögen z.B. den Flugpassagier rasch über das überflogene Gebiet zu orientieren, sie versagen aber in anderer Hinsicht. In den meisten Fällen wünschen wir von der Karte eine sichere Orientierung über die Formen des Reliefs und über die Höhenlagen. Reliefform und Höhenlage sind und bleiben die topographischen Grundelemente. Ihre Darstellung darf nur in Ausnahmefällen einem bunten Farbmosaik geopfert werden. Für allgemeine Gebietskarten kleinen Maßstabes und somit auch für die Länder- und Erdteilkarten des Schweizerischen Mittelschulatlasses ist die zur Anwendung gelangte Art der Geländedarstellung die brauchbarere und klarere; denn sie besitzt folgende Vorzüge:

1. Das schattenpiastische Bild und die Höhenstufenfarben unterstützen sich in idealer Weise. Ein buntscheckiges Mosaik der Landschafstfarben aber würde sowohl den Reliefeindruck wie das Bild der Höhenstufen zerstören.

2. Die Höhenstufen sind genau und eindeutig definiert und abgrenzbar, was für summarische Bodenbedeckungszonen nicht der Fall ist.

3. Den Reliefformen und Höhenlagen der Erdoberfläche kommt im ganzen gesehen und für Karten kleiner Maßstäbe entscheidendere Bedeutung zu als der Oberflächenbedeckung. Die letztere wäre in kleinen Maßstäben infolge ihrer miniaturhaft feingegliederten Aufsplitterung überhaupt nur in stärkster Zusammenfassung darstellbar.

\section{GRAPHISCHE GESTALTUNG UND ANDERES}

Die Ausgabe 1962 des Atlasses erscheint auch äußerlich in einem neuen Gewand. Zeichensprache und Farbtöne der Karten wurden noch konsequenter als bisher den Inhalten angepaßt, um überall möglichst leichte Verständlichkeit zu erreichen.

Großes Gewicht wurde überdies auf eine klare graphisohe Gestaltung aller Buchteile gelegt. Jede überflüssige Belastung der Karten, jede unnötige Rahmenlinie, jedes 
entbehrliche Wort wurden weggelassen und durch verbesserte Anordnung von Titeln und Legenden die Übersichtlichkeit erhöht. Die Kartenblätter wurden in der Höhe um $1,5 \mathrm{~cm}$ reduziert, womit ein handlicheres Buchformat zustande kam.

Die Einleitungstexte mit den Erläuterungen der Signaturen, den Bemerkungen zur Beschriftung, den Ausspracheregeln usw. erfuhren eine Neufassung und Erweiterung. An Stelle des bisherigen langweiligen Schulbuohdeckels trat ein das Auge erfreuender Einband, dessen Reliefbild zum Studium des Werkes einlädt.

\section{DIE HERSTELLUNG DER KARTEN}

Die Herstellung der Karten gliederte sich in drei Stufen:

1. Inhaltliche Bearbeitung und zeichnerische Erstellung der Entwurfsoriginale.

2. Herstellung der Druckoriginale oder, wie sie auch genannt werden können, der Bildträger für die Reproduktion.

3. Kartendruck.

Die Arbeiten der ersten Stufe wurden durch den Verfasser dieser Zeilen und seine Mitarbeiter besorgt, diejenigen der zweiten und dritten Stufe durch die Art. Institut Orell-Füßli AG in Zürich.

\section{Inhaltliche Bearbeitung und zeichnerische Erstellung der Entwurfsoriginale.}

Die Art des Vorgehens ließ sich hier nicht einheitlich normen; denn sie richtete sich nach der Art des Quellenmaterials und nach Inhalt und Form der zu erstellenden Karten. Im Folgenden seien einige typische Fälle beschrieben.

Ein erstes Beispiel: Karte der Volksdichte der Schweiz 1:2000 000, Seite 28.

Die Einwohnerzahlen der einzelnen Gemeinden und Gemeindeteile (Ergebnisse der Volkszählung 1950 des Eidg. Statistischen Amtes) wurden unter Berücksichtigung des Siedlungsbildes der Landeskarte der Schweiz 1:50000 als Mengenpunkte möglichst lagerichtig auf eine topographische Karte 1:200000 resp. auf entsprechende DeckPaus-Papiere aufgetragen, wobei je 200 Personen in einen Punkt, in größeren Städten je 1000 Personen in eine kleine Quadratfläche zusammengefaßt wurden. Anhand dieses angenäherten, sog. absoluten Streuungsbildes und mit Hilfe verschiedener Karten wurden natur- und kulturräumliche Gebiete sinnvoll und einfach abgegrenzt, so daß innerhalb eines Gebietes ungefähr gleiche Dichte der Punktstreuung bestand. Die Planimetrierung all der vielen hundert abgegrenzten Flächenstücke und das Auszählen der etwa 9000 Punkte und Quadrate ergab für jedes Flächenstück die relative Dichte. Diese Dichtewerte wurden nun in neun progressiv wachsende Stufen (siehe Kartenlegende) gruppiert und jedes Fläohenstück der ihm entsprechenden Stufe zugeordnet. Außerdem wurden alle Orte mit über 10000 Einwohnern entsprechend einer aufgestellten Legende eingetragen. Für Orte über 50000 Einwohner deckt sich deren flächenhafte Signatur mit dem Areal der dichtesten Stufe. Der höchste Stufenwert. über 5000 Bewohner auf $1 \mathrm{~km}^{2}$, entspricht der relativen Dichte kompakt überbauter und bewohnter städtischer Areale, so daß hier Stufenareal und Stadtsignatur identisch sind.

Eine verkleinerte Kopie des so entstandenen Vorentwurfes im Maßstab 1:500 000 wurde entsprechend den Legendenfarben bemalt und hierbei alle Abgrenzungslinien zwischen zwei Feldern gleicher Stufe resp. gleicher Farbe eliminiert. Die Konstruktion der Auslandgebiete erfolgte unter Benützung und durch Interpretation aller erreichbaren statistischen und kartographischen Quellen.

Diese große Karte 1:500 000 bildete die inhaltliche Vorlage für die zeichnerische Erstellung der endgültigen Karte. Sie wurde photographisch in den gewünschten Maßstab 1:2000000 reduziert, dann wurden die linearen Elemente mit feinen Federn 
und schwarzer Spezialtusche auf transparente Kunststoff-Folien (Astralon oder ähnliche) gezeichnet. Bei Karten mit linearen Elementen und Signaturen in mehreren Druckfarben (z.B.Skandinavien Wirtschaft 1 und 2, Seite 69) wurde für jede einzelne Druckfarbe ein eigenes solches Schwarz-Original auf Astralon erstellt. Solche Originale sind kopierfähige, einfarbige, lineare und maßgerechte Positive. Ebenfalls auf einen Film und im Maßstab der endgültigen Karte wurde eine Schriftvorlage erstellt zur genauen Angabe der Anordnungen der Namen und aller übrigen Texte. In eine Photokopie des linearen Originals wurden nun die gewünschten Flächenfarbtöne mit Aquarellfarben eingetragen. Für die Erstellung des Mittelschulatlasses standen uns pro Druckbogen 8-10 verschiedene, passend ausgewählte Druckfarben zur Verfügung. Jede Vollfarbfläche einer jeden Druckfarbe kann durch Rasterung aufgehellt (mit dem weißen Papierton gemischt) werden. Wir legten von vornherein fünf verschiedene Rasterstufen fest. Jede Rasterstufe einer jeden Druckfarbe kann mit jeder Rasterstufe einer andern Druckfarbe kombiniert werden. Analog können auch Rasterstufen von drei oder vier Druckfarben kombiniert werden. Aus alledem ergibt sich eine große Auswahl möglicher Farbtöne. Mit Hilfe besonders hergestellter Mustertafeln wählte man nun für jedes einzelne Feld der Karte die passende Farb- und Rasterkombination. Die Ergebnisse solcher Auswahl wurden für jede Karte in einer Farbtabelle zusammengestellt. Solche Farbtabellen erleichterten und sicherten die weitere technische Erstellung, sie waren gleichsam unsere Farbrezepte.

Der hier geschilderte Weg vom Inhaltsentwurf bis zu den ablieferungsbereiten, rein gezeichneten und farbgetrennten Reproduktionsunterlagen wurde für alle thematischen Karten eingeschlagen.

Nach dieser Skizzierung des technischen Ablaufes der Dinge sei kurz auf die beiden entscheidenden Probleme der vorliegenden Volksdichtekartierung hingewiesen. Es sind folgende:

a) Die Abgrenzungen und Größenordnungen der einzelnen Areale oder Mosaikstücke sind von Anfang an dem Endmaßstab und überdies den natur- und kulturräumlichen Gliederungen anzupassen. Hierin aber liegt der schwierigste und unsicherste Teil der Konstruktion. Sollen im Berner Mittelland Areal und Volksmenge einer Anhäufung großer Dörfer (z.B. Langenthal-Herzogenbuchsee) als Dichtezone ausgeschieden oder aber in den umliegenden Großraum mit hinein gerechnet werden? Wie breit soll die besiedelte Talachsenzone in den verschiedenen Alpentälern gewählt werden? Von der Lösung solcher Ermessens- und Maßstabs- und Generalisierungsfragen hängt das Ergebnis wesentlich ab. Eine gute Landeskenntnis ist Voraussetzung, um solche Aufgaben befriedigend lösen zu können.

b) Die Dichtestufenskala ist zweckmäßig zu gliedern. In einer Relativdichtekarte mit Gemeinde- oder Bezirksarealen als Bauelementen des Flächenmosaiks fehlen im allgemeinen die extremen Dichtewerte, weil sich in jedem Areal eine Ortschaft befindet und weil andererseits innerhalb eines jeden Gemeindeareals offene, unbesiedelte Felder liegen. Bei den Arealabgrenzungen nach tatsächlichen Siedlungs- oder Menschenstreuungen, wie sie unsere Karte zeigt, fehlen diese ausgleichenden Momente. $Z$ wischen eng umgrenzten Stadtgebieten und den Hochgebirgseinöden bestehen außerordentliche Dichtegegensätze. Wir wählten daher eine aus neun Stufen bestehende Skala progressiv wachsender Werte, die mit dem Einödenwert $=0-2$ Bewohner pro $1 \mathrm{~km}^{2}$ beginnt und mit der Großstadtkonzentration $=$ über 5000 Einwohner pro $1 \mathrm{~km}^{2}$ endigt. Das im Gebirgsinnern so charakteristische Zusammendrängen der Volksmengen in den größeren Tälern und die Anhäufungen in den flacheren Mündungsgebieten dieser Täler tritt auf diese Weise deutlich in Erscheinung, ebenso die außerordentliche Dichteballung in den Gebieten großer Industrieorte im nördlichen und südlichen Alpenvorland. 
Ein zweites Beispiel: Karten der mittleren Januar-und Juli-Temperaturen der Schweiz 1:2000 000, Seite 20.

Die entsprechenden Karten der früheren Ausgaben des Mittelschulatlasses gingen auf Entwürfe zurück, die um das Jahr 1900 konstruiert worden waren. Ihr Inhalt war zu stark generalisiert, so daß z.B. in der Januarkarte die Ebene von Meiringen mit unter $600 \mathrm{~m}$ Meereshöhe und die Gebirgskette zwischen Entlebuch und Sarnersee mit Höhen von über $2000 \mathrm{~m}$ in ein und derselben Wärmestufe lagen.

Eine Neukonstruktion drängte sich auf. Diese stützte sich auf die Berichte, Veröffentlichungen und Tabellen der Schweizerischen Meteorologischen Zentralanstalt, des Deutschen Wetterdienstes, des Hydrologischen Dienstes in Österreich, der Ente nazionale industrie turistiche (Italien) und für Frankreich auf J. Sanson: Recueil de données statistiques relatives à la climatologie de la France, Paris 1945. Für die Schweiz standen uns die Monatsmittel der Temperaturen für Januar und Juli der Beobachtungsperiode 1901-1940 von 90 Wetterstationen zur Verfügung, für das ausländische Kartengebiet die Werte von insgesamt 200 Stationen, zum Teil ebenfails für die Periode 1901-1940, zum Teil für diejenige von 1901-1950. Diese Werte aber reichten nicht aus zur Kartenkonstruktion. Die Daten mußten verdichtet werden mit Hilfe der mehr oder weniger gesetzmäßigen Beziehungen zwischen Höhenänderung und Änderung der Mitteltemperaturen. Auf Grund lokaler Temperaturmittel und beraten durch die Schweizerische Meteorologische Zentralanstalt konstruierten wir für einige sinnvoll ausgewählte Gebiete die mittleren vertikalen Temperaturgradienten, sowohl für den Januar wie für den Juli. Es ergaben sich folgende Werte:

$\begin{array}{lcc}\text { Höhenlage } & \begin{array}{c}\text { Vertikaler Temperaturgradient } \\ \text { (Mittlere Abnahme der Temperatur } \\ \text { pro } 100 \mathrm{~m} \text { Höhenzunahme) }\end{array} \\ & \text { Januar } & \text { Juli } \\ \text { Unter } 1500 \mathrm{~m} & 0,4^{0}-0,5^{\circ} & 0,5^{\circ}-0,6^{\circ} \\ \text { Über } 1500 \mathrm{~m} & 0,5^{0} & 0,6^{\circ}\end{array}$

Mit Hilfe dieser Werte und der Meßergebnisse der Wetterstationen konnten nun auf der Basis einer Höhenstufenkarte der Schweiz im Maßstab 1:1000 000 die Isothermen konstruiert und im Einzelnen dem Geländerelief angepaßt werden. Das Weitere war auch hier wieder Verkleinerungs-, Generalisierungs- und Kontrollarbeit und darauf Reinzeichnung, wie wir es oben für die Volksdichtekarte beschrieben haben.

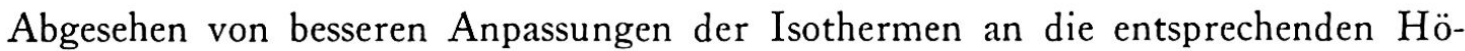
henlinien unterscheiden sich unsere beiden neuen Karten der Periode 1901-1940 von denjenigen der Periode 1864-1900 im bisherigen Atlas vor allem durch folgendes:

Die neue Karte der Januar-Temperaturen läßt erkennen, daß auf der Alpennordseite, gegenüber der früheren Beobachtungsperiode eine Erwärmung um etwa $0,7^{\circ}$ eingetreten ist. Das Wärmegebiet des Genfersees (über $0^{\circ}$ ) dehnt sich nun aus bis zum Neuenburgersee und Bielersee. Auch die Zone zwischen $-2^{\circ}$ und $0^{\circ}$ im Mittelland (Bern - Luzern - St. Gallen) ist ausgedehnter als früher. Ein Vergleichen der neuen mit der früheren Julikarte zeigt eine gegenteilige Erscheinung: Auf der Alpennordseite eine Abkühlung von etwa $1^{\circ}$, auf der Alpensüdseite eine solche von etwa $0,5^{\circ}$.

Kleine lokale Wärme- oder Kältebecken treten in den Januar- und Julimittelwerten kaum in Erscheinung. Hingegen ist in der Januarkarte der Einfluß des Föhns erkennbar aus einigen angeschriebenen Stationswerten (Altdorf $=+0,5^{\circ}$ und Luzern bei gleicher Höhenlage $=-0,2^{\circ}$; Interlaken $=-0,4^{\circ}$ und das tiefer gelegene Bern $\left.=-1,0^{\circ}\right)$. 
Ein drittes Beispiel: Karte der Niederschläge der Schweiz 1:2000 000, Seite 22.

Die oben besprochenen Beispiele zeigten komplizierte Fälle, völlige Neubearbeitungen auf Grund lokaler Zählungen und Messungen. Die Herstellung unserer Karte der Niederschläge der Schweiz repräsentiert das andere Extrem, den einfachsten Fall. Hier bestand bereits eine neue und einwandfreie Karte, die «Niederschlagskarte der Schweiz» 1:500 000 (Periode 1901-1940), von H. UtTinger, Schweiz. Meteorologische Zentralanstalt (Verlag: Schweizerischer Wasserwirtschaftsverband, Zürich 1948). Die Bearbeitung für den Schweizerischen Mittelschulatlas beschränkte sich hier auf die Wahl der dem neuen, kleineren Maßstab und den Atlasnormen angepaßten Stufen und Stufenfarben, auf die linearen Vereinfachungen, auf die Auswahl der orientierenden Flüsse und Orte.

$Z$ wischen den Fällen des ersten, zweiten und dritten Beispieles lagen, je nach Quellenmaterial und geplantem Karteninhalt, bald einfachere, bald kompliziertere Fälle.

\section{Ein viertes Beispiel: Karte von Ostasien 1:15000 000, Seite 90-91.}

Völlig verschieden von den Bearbeitungen thematischer Karten waren diejenigen der allgemeinen topographischen Gebiets- oder Länderkarten, wie z. B. derjenigen von Ostasien. Hier bestand eine Hauptschwierigkeit in der Beschaffung genügenden und genügend neuen kartographischen und statistischen Quellenmaterials. Wir haben auf diese Sorge des Atlaserstellers in einem vorangehenden Abschnitt bereits hingewiesen. Wir zeichneten alle Karten solcher Art aus Genauigkeitsgründen in (linear) 11/2facher Größe: zuerst Konstruktion des Kartennetzes, dann, nach Druckfarben getrennt, auf je einer Transparentfolie, die Zeichnung des Gewässernetzes, der linearen Situation inklusive Ortssignaturen, des Bahnnetzes, der politischen Grenzlinien, der Kartenbeschriftung. Hierbei handelte es sich im wesentlichen um Generalisierungsaufgaben. Die beigezogenen Grundlagekarten enthalten eine Fülle von Objekten, deren Eintragung in einer Schulkarte kleineren Maßstabes weder angebracht noch überhaupt möglich wäre. Es ist gar nicht leicht, eine angemessene Auswahl der Objekte, Namen und Koten zu treffen; denn es rücken immer wieder neue Gebiete und Dinge in das Blickfeld des öffentlichen und des geographischen Interesses. Zweckmäßiges Auswählen und gutes lineares Vereinfachen setzen beim ausführenden Kartographen viel geographisches Wissen und graphisches Können voraus. Das Gewässernetz, ergänzt durch positive und negative Reliefkanten, wurde als ausfixierbare Blaukopie oder Bromsilberkopie auf ein möglichst weißes, glattes Papier gebracht. Um jegliches Verziehen zu vermeiden, müssen solche Papiere auf dünne Aluminiumplatten aufgeleimt sein. Auf dieser Papier-Aluminium-Folie wurde mittels harter, scharfer Qualitätsbleistifte, schwarzer Aquarellfarbenmalerei und mit Hilfe des sogenannten «Luftpinsels» das Relieforiginal als ein von Weiß bis Schwarz abgetöntes Bild erstellt. Der Luftpinsel, auch bekannt unter den Bezeichnungen «Air-Brush» und «Spritzpistole», ist ein Gerät, mit welchem ein äußerst feiner Strahl zerstäubter flüssiger Farbe auf das Papier geschossen werden kann. Erst auf Grund des so erstellten Relieforiginalbildes konnte dann eine lineare und durch Farbflächen verdeutlichte Vorlage der farbigen Höhenstufen erstellt werden. Die Stufenfarben und ihre Zusammensetzungen aus den verschiedenen Druckfarben und Rastern wurden hierauf in Tabellen nochmals festgehalten. (Siehe darüber Lit. Nr. 4).

Auch für jede solche Länderkarte wurde auf einer transparenten Folie eine Schriftvorlage mit lage- und formgerechter Eintragung jedes Namens und jeder Höhenkote erstellt. Dieser Schriftvorlage wurde ein Namensverzeichnis sowohl für die deutsche wie für die französische und italienische Atlasausgabe beigegeben. In diesen Verzeichnissen wurden die Namen gruppiert nach Schriftarten, Schriftgrößen usw. Beispielsweise enthielt das Namenverzeichnis für die 1 1 $\frac{1}{2}$ seitige Ostasienkarte (deutsche Ausgabe) 320 Namen und 100 Höhenkoten. 


\section{Ein füntes Beispiel: Karte des Mount Everest 1:100 000, Seite 93.}

Herstellungstechnisch wiederum ein sehr einfaches Beispiel, da hierfür als Quelle nur eine einzige Karte in Frage kam: die neue, vorzügliche Karte des Mount Everest 1:25 000, aufgenommen von ERwIN SchNeIDER und herausgegeben 1957 vom Deutschen Alpenverein, vom Österreichischen Alpenverein und von der Deutschen Forschungsgemeinschaft.

Für unsere neue Karte war der Maßstab 1:100 000 gewählt worden, um es damit dem Schüler zu ermöglichen, die Dimensionen asiatischer Bergriesen mit den schweizerischen Bergen in den Karten 1:100 000 der Seiten 6 und 7 (Jungfraugruppe, Berninagruppe) vergleichen zu können. Auch die Darstellungsart ist dieselbe. Es handelte sich somit hier für uns lediglich um eine Generalisierungs- und Darstellungsaufgabe. Auf Grund der genannten Karte 1:25000 wurde auf einem transparenten Blatt eine Kopie der für unsere kleinere Karte wesentlichen linearen Elemente erstellt, dann dieser «Stoffauszug» photographisch auf den Maßstab 1:100 000 reduziert, hierauf das Bild der Felsen, Gerölle, Moränen usw. in stark vereinfachter und plastisch erscheinender Form neu mit der Feder auf Astralon gezeichnet und schließlich die Höhenkurven und Bachlinien durch Schichtgravur mit der Nadel erstellt. Darauf wurden die farbgleichen Elemente zusammenkopiert, Schriftvorlage und Schriftfilm erstellt und schließlich auf der Basis einer ausfixierbaren Blaukopie aller linearen Elemente ein schwarz-weiß abgetöntes Relieforiginal erstellt. Die Farbtöne wurden durch besondere Vorlagen und Instruktionen festgelegt.

Diese Karte zeigt erstmals in der schweizerischen Kartenmanier einen kleinen Ausschnitt aus dem höchsten Gebirge der Erde. Die Verwandtschaft der Formen, der steilwandigen Grate und flachen Firnkare, der Eisströme und Moränen mit denjenigen der Alpen ist in die Augen springend. Es sind die Formen des glazial umgeformten Hochgebirges. Bei genauerer Betrachtung aber zeigen sich gegenüber den Alpen einige Unterschiede: Das gesamte Grundgestell dieses Gebirges ist 3-4000 m höher als in den Alpen, die Gangesebene als Erosionsbasis liegt sehr tief. Die Temperaturunterschiede von Tag und Nacht sind hier, in unmittelbarer Nähe der Tropen, viel größer als bei uns. Der Monsun überschüttet das Gebirge mit enormen Niederschlägen. Die Erosionskräfte sind verstärkt, die Karrückwände bedeutend steiler und höher als in den Alpen. Die großartigen Tal- oder Zungengletscher fließen rasch in tiefe Regionen und sind daher an den Südabdachungen des Himalaya nicht länger als die größten Gletscher der Alpen, doch sind sie infolge der intensiveren Hangverwitterung äußerst schuttreich. Sie kriechen daher zwischen hohen Seitenmoränen dahin und liegen aufgebahrt in enormen Schuttsärgen.

Die geschilderte erste Arbeitsstufe führte für jede einzelne der 172 neuen Karten zur Erstellung folgender 5-10 Dokumente durch den Atlasredaktor:

1-4 lineare Originalvorlagen auf Film (je eine für jede Druckfarbe)

1 Farbflächenoriginal auf Papierkopie

1 Farbtabelle

1 Schriftoriginal auf Film

1 Namenverzeichnis: deutsch, französisch und italienisch

Für die allgemeinen Gebiets- und Länderkarten und Erdteilübersichtskarten waren ferner zu erstellen:

1 Relieforiginal in $11 / 2$ facher Vergrößerung auf Papier (Aluminiumfolie)

1 lineare Höhestufenvorlage auf Film

Dazu kamen zahlreiche Korrekturvorlagen für die nur revidierten, nicht neu entworfenen Karten. Für jede Kartenseite war überdies erforderlich 1 Vorlage für die Anordnung und Form der Karten, ihrer Titel und Legenden (in deutscher, französischer und italienischer Sprache). 


\section{Herstellung der Druckoriginale oder Reproduktionsbildträger.}

Auch hier betrachten wir wiederum zunächst die Volksdichtekarte der Schweiz $1: 2000000$, Seite 28 .

Feine lineare Bildelemente, wie z.B.das Gewässernetz und die Farbflächenkonturen, wurden in einigen Fällen schon bei der Erstellung der Entwurfsoriginale mit Feder und Tusche vergrößert und so sorgfältig gezeichnet, daß ihre photographische Verkleinerung und anschließende Umkopie auf transparente Folie unmittelbar die Druckoriginale (Reproduktionsbildträger) ergaben. In den meisten Fällen aber gelangte das folgende Verfahren zur Anwendung: Man erstellte das lineare Original nur als Entwurfszeichnung, photokopierte diese auf eine mit einer lichtundurchlässigen Gravurschicht bedeckte Kunststoffolie, worauf die entsprechende Schichtgravur mit Gravurgeräten ausgeführt wurde. In gewissen Fällen werden beschichtete Glasplatten den Kunststoffolien vorgezogen. Das Schichtgravieren mit Spezialgeräten geht in der Regel wesentlich rascher als das Zeichnen mit Federn, und es führt zu besserer Bildqualität.

Die Druckoriginale für die verschiedenen Flächenfarben wurden auf folgende Art erstellt: Für jede Rasterstufe einer jeden Druckfarbe wurde eine entsprechende Volltonmaske erstellt, die entweder durch Decken mit schwarzer Tusche oder durch das sogenannte Strippen entstand. Mit Hilfe dieser Masken erhielt man das Schwarzweißoriginal jeder zu druckenden Farbe auf einer transparenten Folie, indem eine Stufe nach der anderen mit je einem Kontaktlinien- oder Punktraster verschiedener Tonwirkungen ineinander kopiert wurde.

Die Beschriftungsd ruckoriginale wurden erstellt durch Zusammenfügen von eigens hierfür hergestellten negativen Buchstabentäfelchen in Setzrahmen. Durch photographische Verkleinerung entstand direkt ein Positiv auf Stripping-Film. Die einzelnen Namen wurden ausgeschnitten, auf eine transparente Folie lagerichtig montiert und nachher auf den endgültigen Originalbildträger umkopiert. Als Vorlagen für dieses technische Erstellen der Schriftfilme dienten die obengenannten Beschriftungsentwürfe und Namenverzeichnisse.

Zur Reproduktion der Geländerelieftöne der Länderkarten waren pro Karte zwei in den Tonwerten verschiedene Aufnahmen erforderlich. Die erste gibt das Original möglichst genau wieder, während die zweite - eine sogenannte «harte» Aufnahme nur noch die kräftigsten Schattentöne aufweist. (Näheres darüber siehe Lit. Nr. 4).

Diese Arbeiten bei Orell-Füßli umfaßten eine große Menge äußerst exakter Gravur-, Zeichnungs-, Retouchier- und Montagearbeiten, ferner photographische Präzisionsaufnahmen und Kopierprozesse. Für jede einzelne der ca. 250 Karten des Atlasses ergaben sich je ein Film für jede der 6-10 oder im Mittel etwa 8 Druckfarben und zusätzlich je ein Film für die französische und italienische Beschriftung, somit pro Karte etwa 10 Filme. Für die ca. 250 Karten waren somit etwa 2500 Filme herzustellen.

Die Operate der einzelnen Karten mußten jeweils zu ganzen Druckbogen zu je 8 Seiten (Vorder- und Rückseite zusammengerechnet) zusammengefügt werden. Für jeden der 18 Bogen ergaben sich hieraus folgende Operate: ein zusammenkopierter Film für jede der 8-10 oder im Mittel $=9$ Druckfarben, und zwar je für die Vorder- und Rückseite des Bogens, somit pro Druckbogen $=9 \times 2=18$ Filme. Dazu kamen zusätzlich je ein Film für die französische und italienische Beschriftung, ebenfalls für die Vorder- und Rückseite des Bogens, somit $2 \times 2=4$ Filme. Dies ergab insgesamt pro Druckbogen $=18+4=22$ Filme oder für alle 18 Druckbogen zusammen $18 \times 22=396$ Filme.

Jeder dieser 396 Filme war auf eine Maschinenplatte (Druckplatte) zu kopieren. 
Wir fassen zusammen:

Aus den oben besprochenen Arbeitsgängen ergaben sich für jede einzelne Karte etwa 5-10 redaktionell-zeichnerische Vorlagedokumente oder insgesamt für $250 \mathrm{Kar}$ ten annähernd 2000 solche Operate.

Zur Erstellung der farbgetrennten Bildträger durch Orell-Füßli waren im Mittel pro Atlasseite etwa 30 einfache und kombinierte Filme mit 40 Kopiervorgängen erforderlich. Dies führte für 144 Seiten zu $30 \times 144=4320$ Filmen mit etwa $40 \times 144$ $=5760$ Kopiervorgängen. Dazu kam die Erstellung der Maschinenplatten, was für 18 beidseitig mit je 9 Farben zu bedruckenden Bogen und den zusätzlichen Platten für die französische und italienische Beschriftung zu 396 weiteren Kopiervorgängen führte. Die Atlaserstellung erforderte somit insgesamt $5760+396=6156$ Kopiervorgänge.

\section{Der Kartendruck}

Vor Beginn des Auflagedruckes der Karten wurden Probedrucke der einzelnen Atlasbogen zur Kontrolle aller Inhaltsteile und der Farbwahl erstellt. In den meisten Fällen zeigten erst diese Zusammendrucke aller Farben dem Kartenredaktor erstmals das fertige, inhaltsvereinigte, farbige Kartenbild. Erst hier zeigte es sich, ob er seine Dispositionen zweckmäßig getroffen, die Farbtabellen richtig aufgestellt, das stets so entscheidende Zusammenspiel aller Elemente gut disponiert hat. Das Eintreffen eines Probedruckes war daher stets ein Augenblick höchster Spannung. Im Probedruck werden die auf den Druckoriginalen noch anzubringenden Korrekturen, Ergänzungen und Streichungen eingetragen. Entgegen häufigem Gebrauch beschränkten wir uns beim Mittelschulatlas in der Regel pro Druckbogen auf ein nur einmaliges Erstellen von Probedrucken. Einige eventuell nicht behobene Fehler wiegen in den meisten Fällen leichter als eine Buchverteuerung, die durch weitere Probedrucke verursacht worden wäre. Heute werden Probedrucke oft ersetzt durch photographische Vielfarbenkopien (Multicolorkopien).

Der Auflagedruck des Atlasses erfolgte auf $Z$ weifarben-Offsetpressen. Jeder der 18 Druckbogen enthält auf der Vorder- und Rückseite je 4 passend zusammengestellte Atlasseiten. Zum Druck jedes Bogens waren im Mittel 9 Druckfarben erforderlich. Somit ergaben sich für Vorder- und Rückseite eines jeden Bogens 18 Druckgänge. Dies machte für 18 Bogen bei einer Auflage von 50000 Stück $=18 \times 18 \times 50000=$ 16200000 Druckgänge, was den Einsatz einer Zweifarben-Offsetschnellpresse während $1 \frac{1}{2}$ Jahren erforderlich machte. Die Druckbogen für die deutsche, französische und italienische Ausgabe sind, mit Ausnahme des Schriftbildes, identisch, so daß sie zusammen gedruckt werden konnten. Die Kartenbeschriftung sitzt für jede der genannten drei Sprachen auf einer eigenen Platte, sie wurde separat in die betreffenden Auflageteile hineingedruckt. Nur so war es möglich, eine relativ kleine italienische Auflage des Atlasses für unsere Tessiner Schulen zu relativ bescheidenem Preise herzustellen.

Aus diesen Darlegungen möge hervorgehen, wie grundlegend sich die kartographische Reproduktionstechnik in jüngster Zeit gewandelt hat. Die einstigen Kupferstichplatten und die Lithographiesteine sind heute aus modernen Kartenreproduktionsanstalten verschwunden. Sie waren schwerfällig und nicht leicht nachführbar. Der Kupferstich, die lithographische Fettzeichnung mit Feder, Tusche und Kreide und die sog. «Steingravur» sind durch die dargelegten neuen Verfahren verdrängt worden. Auch das umständliche «Umdrucken» ist durch das einfachere Kopieren ersetzt. Dies alles hat zu beträchtlichen Vereinfachungen, Beschleunigungen und auch zu Qualitätsverbesserungen geführt. Vor allem aber sind mittels der neuen Verfahren nachträgliche Inhalts- und Farbänderungen, Nachträge und Korrekturen leichter und rascher anzubringen, als es früher der Fall war. Diese Neuerungen kamen nun auch dem Schweizerischen Mittelschulatlas zugute. 
Bei all diesen Vorgängen, bei jedem Federstrich, bei der Gravur, bei der Kopierund Montagearbeit und beim Drucken sind höchstmögliche Lage- und Einpaßgenauigkeiten einzuhalten. Daß dies bei solch großem Gesamtarbeitsvolumen gelang, danken wir dem nimmermüden Einsatz all der vielen «unbekannten Soldaten» in unsern kartographischen Betrieben. Als ein reproduktionstechnisches Erzeugnis bildet der Schweizerische Mittelschulatlas in seiner neuen Gestalt ein hervorragendes Zeugnis schweizerischer Qualitätsarbeit.

\section{DIE MITARBEITER}

Außer den Angestellten des Atlasredaktionsbüros in Erlenbach-Zürich und denjenigen der Art. Institut Orell-Füßli AG in Zürich wirkten bei der Bearbeitung einzelner Karten eine Reihe weiterer Fachleute mit. Wir nennen sie im Folgenden gruppiert nach den Karten oder Kartengattungen des Atlasses. Soweit es sich um Karten handelt, die im wesentlichen aus früheren Auflagen stammen, führen wir auch deren einstige Bearbeiter hier auf.

Schweiz Tektonik (S. 16) und Geologie (S.17), sowie Alpenländer Geologie (S. 26-27) : Prof. Dr. J. Cadisch, Bern, unter Mitarbeit von Dr. K. Arbenz, jun., Bern, für die Atlasauflage 1948. Beratende Mithilfe für die Atlasausgabe 1962 wiederum Prof. Dr. J. Cadisch, ferner Prof. Dr. A. Gansser, Zürich, und Prof. Dr. E. DAL Vesco, Zürich. Bodenschätze und Ausbeutungsstellen in der geologischen Karte der Schweiz: Prof. Dr. F. DE Quervain, Zürich.

Klimatologische Karten: Dr. M. Sснüepp, Zürich, bearbeitete die Karte S. 21 der typischen Wetterlagen und half beratend und durch Beschaffung der statistischen Daten mit bei der Erstellung der Karten der mittleren Januar- und Julitemperaturen der Schweiz (S. 20). Die Konstruktion der letztgenannten Karten erfolgte gemeinsam mit dem Redaktor durch A. Dürst, Dipl. Geograph, Zürich.

Schweiz.Volksdichte (S. 28): Vorbereitende Konstruktionen (Punktkarte): C. Aubert, Dipl. Naturwissenschafter, Genf.

Schweiz, Zu- und Abnahme der Bevölkerung (S.28): Entwurf: Prof. Dr. J. HösLI, Männedorf, für die Atlasausgabe 1948.

Vegetationskarten der Erdteile und der Erde (S. 77, 85, 102, 113, 122, 129, 137): Entwurf: Prof. Dr. Emil Schmid, Zürich, für die Atlasausgabe 1948.

Völker- und Religionskarten der Erdteile und der Erde (S. 89, 107, 127, 140): Verschiedene frühere Bearbeiter und Quellen. Revision durch M. WEHRLI, Bern, A. Dürst, Zürich, und den Redaktor.

Wirtschaftsgeographische Karten: Die folgenden Karten dieser Gruppe wurden für die Ausgabe 1948 durch Prof. Dr. J. Hösli, Männedorf, in Gemeinschaft mit dem Redaktor, bearbeitet: Schweiz. Industrie und Gewerbe (S.22), Schweiz. Landwirtschafts- und Industriegebiete (S. 23), Frankreich, Mitteleuropa, Italien und Balkan: Landwirtschaft und Fischerei, Bergbau und Industrie (S. 34-35, 46-47, 60), ferner Iberische Halbinsel Bergbau und Industrie (S.61), Britische Inseln: Landwirtschaft, Fischerei, Bergbau, Industrie (S. 64), ferner die Wirtschaftsübersichtskarten der Erdteile und der Erde (S. 83, 92, 106, 116, 122, 129, 139), die Produktekarten von Afrika, Nordamerika, Südamerika und Australien (S. 106, 116-117, 123, 129). Bei der Bearbeitung einiger dieser Karten wirkte auch Prof. Dr. H. Boesch beratend mit. Für die Atlasausgabe 1962 wurden einige Vereinfachungen und Anpassungen an jüngere Quellen durch den Redaktor vorgenommen.

Erdübersichten der Produkte (S. 140-141): Neukonstruktionen durch A. DürsT, Zürich, in Zusammenarbeit mit dem Redaktor und beraten durch Prof. Dr. H. Boesch, Zürich. 
Die in der Atlasausgabe 1962 erstmals auftretenden wirtschaftsgeographischen Karten (Skandinavien, Asien usw.) wurden durch den Redaktor bearbeitet.

Besonders hervorgehoben seien hier die Kartenkünstler meines Ateliers in Erlenbach-Zürich, die in mühsamer Kleinarbeit, aber mit größtem Geschick die neuen Relieforiginale der Länder- und Erdteilkarten zeichneten. Es waren H. LEUBNER, jetzt Stuttgart, H. Leuzinger, Herrliberg-Zürich, G. Pöhlmann, jetzt in Berlin-West, W. Rufener, jetzt Primarlehrer in Spiez, und B. Speiser, Zürich.

Als vorzügliche Gehilfin beim graphischen Gestalten sei genannt Fräulein H. LASER, Erlenbach-Zürich, als Mitwirkende bei mancherlei Entwürfen und bei der Quellenbeschaffung Fräulein H. Kutzsch, Zürich, und A. Dürst, Zürich, vor allem aber meine Frau V. ImHof-MAY.

Die Texte und Namen in den Karten der früheren Atlasausgaben waren durch Prof. Dr. CH. Burky, Genf, überprüft und bereinigt worden. Für die Ausgabe 1962 wurden diese Arbeiten besorgt durch Fräulein B. Ruedin, Bern. Die entsprechenden Arbeiten der italienischen Ausgaben besorgte seit vielen Jahren Prof. Dr. E. DAL Vesco, Zürich.

Die kartographisch-technischen Mitarbeiter der Anstalt Orell-Füßli waren so zahlreich, daß wir leider auf die Nennung ihrer Namen verzichten müssen. Besonders hervorgehoben seien jedoch R. BARTHEL, Zürich, der vor 1957 die kartentechnischen Arbeiten leitete, und sein Nachfolger W. BANTEL, seit 1957 Leiter der Kartographischen Abteilung von Orell-Füßli. Diesem letzteren kommt ein Hauptverdienst am Gelingen des neuen Werkes zu.

Es sei an dieser Stelle allen hier genannten und auch den nicht genannten Mitarbeitern, Helfern und Beratern der beste Dank des Atlasredaktors ausgesprochen. Besonderer Dank der Öffentlichkeit und der Schule gebührt der Konferenz der kantonalen Erziehungsdirektoren und deren Atlasdelegation, ferner den Eidg. Departementen des Innern und der Finanzen; denn es bedurfte zur Realisierung der Atlasumformung deren besonderes Verständnis und Wohlwollen für schulgeographische und kartographische Anliegen.

\section{LITERATUR}

1. HösLI, J.: Neue wirtschaftsgeographische Karten des Schweizerischen Mittelschulatlasses. In: Geographica Helvetica, Bern. Jg.3, 1948. S. 393-4o4. 2. ImнoF, E.: Der Schweizerische Mittelschulatalas. Geographica Helvetica, Bern Jg.3, 1948. 82 S., 13 Abb., 4 Karten; 3. - Der Schweizerische Mittelschulatlas und die an unseren Schulen verwendeten Unterstufenatlanten. Archiv für das schweizerische Unterrichtswesen. 43. Jahrgang, Frauenfeld 1957. (Mit weiteren Literaturangaben) ; 4. - Eine neue Karte der Alpenländer, Geographica Helvetica. Bern, Jg. 1959. S. 65-75 mit einer Karte; 5. - Reliefdarstellung in Karten kleiner Maßstäbe. Internationales Jahrbuch für Kartographie. Jg.1. Gütersloh und Zürich, 1961. Mit 1 Karte. 6. Keates, J. S.: The small-scale representation of the landscape in colour. Internationales Jahrbuch für Kartographie. Jg. II. Gütersloh und Zürich, 1962. Mit 1 Karte. 7. Lersch, E.: Begleitworte zur 6. Auflage (1932) des Schweizerischen Mittelschulatlasses. Basel 1934. 8. ScHMid, E.: Die Vegetationskarten im Schweizerischen Mittelschulatlas, Geographica Helvetica, Bern. Jg. 3, 1948, S. 385-392. 9. SchüвpP, M.: Erläuterungen zu den Karten typischer Wetterlagen im Schweizerischen Mittelschulatlas. Geographica Helvetica. Bern. Jg. 1948. S. 376-384.

\section{RÉ S U MÉ}

Au printemps 1962 , l'«Atlas scolaire suisse pour l'enseignement secondaire» a paru sous une forme nouvelle. Son rédacteur, M. EDuARD IMHOF, donne un compte-rendu du contenu, du genre des sources. La nouvelle réalisation graphique est expliquée, en particulier la nouvelle présentation du relief. Quelques exemples démontrent la rédaction et le dessin, et certaines explications concernant la technique de reproduction sont données. Finalement, les principaux collaborateurs sont cités. 